University of Wollongong

Research Online

Faculty of Business - Papers (Archive)

Faculty of Business and Law

$1-1-2019$

Children and "junk food" advertising: Critique of a recent Australian study

John R. Rossiter

University of Wollongong, jrossite@uow.edu.au

Follow this and additional works at: https://ro.uow.edu.au/buspapers

Part of the Business Commons

Research Online is the open access institutional repository for the University of Wollongong. For further information contact the UOW Library: research-pubs@uow.edu.au 


\title{
Children and "junk food" advertising: Critique of a recent Australian study
}

\begin{abstract}
A field experiment by Australian public health researchers Norman, Kelly, McMahon, and others allegedly demonstrated that primary school children's physical health is threatened by exposure to "junk food" advertising. Their study was published in the international health journal Appetite and was clearly intended to influence government regulatory policy. The methodology of the study was taken, without acknowledgement, from an earlier American study in the Journal of Consumer Research and much other research on children's consumer behavior, including pioneering studies conducted by the present author, was ignored. Also, quite unforgivably, the researchers neglected to cite Australia's already strict regulatory controls on and guidelines for advertising directed to children. The present author sent a rejoinder to Appetite pointing out the many problems with Norman et al.'s research and recommending that their article be withdrawn, but the editor insisted I revise it to remove almost all the criticisms, which I naturally refused to do. Instead, by publishing this article in JCB, the present author is hoping to make consumer researchers aware of the sort of poorly performed and ideologically influenced research relating to marketing and consumer behavior that is appearing in the health journals. The present article also contributes more generally by exposing problems that must be avoided in experimental research on consumer behavior.

Disciplines

Business

Publication Details

Rossiter, J. R. (2019). Children and "junk food" advertising: Critique of a recent Australian study. Journal of Consumer Behaviour: an international research review, 18 (4), 275-282.
\end{abstract}

This journal article is available at Research Online: https://ro.uow.edu.au/buspapers/1593 


\title{
Children and “junk food” advertising: Critique of a recent Australian study
}

\author{
John R. Rossiter ${ }^{1,2}$ \\ ${ }^{1}$ Faculty of Business, University of Wollongong, NSW, Australia \\ ${ }^{2}$ School of Psychology, Charles Sturt University, NSW, Australia \\ Correspondence: Professor John R. Rossiter, Level 2, Mike Codd Building, Innovation \\ Campus, University of Wollongong, Wollongong NSW 2522, Australia. Email: \\ john_rossiter@uow.edu.au
}

\begin{abstract}
A field experiment by Australian public health researchers Norman, Kelly, McMahon, and others allegedly demonstrated that primary school children's physical health is threatened by exposure to "junk food" advertising. Their study was published in the international health journal, Appetite, and was clearly intended to influence government regulatory policy. The methodology of the study was taken, without acknowledgement, from an earlier American study in the Journal of Consumer Research and much other research on children's consumer behavior, including pioneering studies conducted by the present author, was ignored. Also, quite unforgivably, the researchers neglected to cite Australia's already strict regulatory controls on, and guidelines for, advertising directed to children. The present author sent a rejoinder to Appetite pointing out the many problems with Norman et al.'s research and recommending that their article be withdrawn, but the editor insisted I revise it to remove almost all the criticisms, which I naturally refused to do. Instead, by publishing this article in $J C B$ the present author is hoping to make consumer researchers aware of the sort of poorly performed and ideologically influenced research relating to marketing and consumer behavior that is appearing in the health journals. The present article also contributes more generally by exposing problems that must be avoided in experimental research on consumer behavior.
\end{abstract}

\section{Keywords}

children and food advertising, research ethics, research mistakes, low-quality vetting of policy-relevant research 


\section{Children and “junk-food” advertising: Critique of a recent Australian study}

\section{INTRODUCTION}

A major issue being debated at present in Australia is whether so-called junk-food advertising aimed at children should be banned in light of the well-publicized finding that approximately one in four Australian schoolchildren is now overweight or obese (Griscti, 2018; Watkins, 2018). Important policy decisions such as this obviously must be based on good research evidence. Recently, evidence in support of a ban on "unhealthy food" advertising directed to children was put forward in Appetite by Australian researchers Norman, Kelly, McMahon, Boyland, Baur, Chapman, King, Hughes, and Bauman (2018a) in a government-funded study clearly designed to influence regulatory policy. This team of public health academics and government health researchers, working together on a project funded by the Australian Research Council and the Cancer Council of the state of New South Wales, conducted an experiment with Australian children attending a holiday camp at which the children were exposed to what the researchers considered to be advertising for "junk food" on some days and to non-food advertising on others and then were invited to choose from a range of allegedly healthy and unhealthy snack foods immediately afterward. The children seemed to eat more calories of snacks on the junk-food advertising days than on the non-food advertising days, leading the researchers to conclude (p. 442) that Australia needs a "more stringent regulatory policy to restrict children's exposure to unhealthy food marketing..." Norman et al.'s study was reported favorably in the major national newspaper, The Australian (see Parnell, 2018), which is important because news reports are likely to be the only "evidence" that policymakers will see.

But does Norman et al.'s study provide good evidence for the alleged harm resulting from children's exposure to junk-food advertising? It is the aim of the present article to demonstrate, by exposing serious problems with the research design, methodology, and analysis, that Norman et al.'s research is not valid and so the findings cannot be trusted, and that their study cannot be used to either shape or justify regulatory policy. These problems point to the need for much more thorough vetting of research proposals before the research begins so as to prevent research mistakes such as those found in the Appetite study.

\section{NORMAN ET AL.'S STUDY}

The study was conducted by first author Jennifer Norman, apparently as her $\mathrm{PhD}$ thesis in public health supervised by professors Kelly and McMahon at the University of Wollongong, and experienced senior researchers in public health, professors Baur and Bauman of the University of Sydney, served as advisors (see all authors' research contributions as stated on p. 443 of their article).

In the study, 160 primary school children, ranging from 7 to 12 years of age, were invited to attend one of four 6-day camps, $n=40$ children per camp, that ran from 8 a.m. to 1:30 p.m. during school holidays. Shortly before morning snack time each day, the children were exposed to advertising media. Half the children attending each camp were randomly allocated to either a TV advertising group, in which TV commercials were embedded in a children's cartoon, or a group in which the same cartoon containing TV advertising was followed by playing a computer game that featured a branded product throughout. 
A within-group crossover and counterbalanced design was used. For the first three mornings of the camp, the children were exposed to advertising for food products deemed by the researchers, according to the nutrient profiling criteria developed by Food Standards Australia New Zealand in 2015, to be "unhealthy," and thus "junk food.” For the second three mornings of the camp, the children were crossed over by exposing them to advertising for non-food products. This sequence of exposure, three days of junk-food advertising followed by three days of non-food advertising, was reversed in two of the four camps to form a counterbalanced design.

At morning snack time, immediately after exposure to either the junk-food advertising or the non-food advertising, each child was allowed to choose items freely from six 50-gram $(12 / 3$ oz.) bowls of snack food containing, respectively, snacks described in the article as "high-fat savory," "low-fat savory," "high-fat sweet," "low-fat sweet," "fruit," and "vegetables" (p. 440). The children were told by the researcher that they could eat "as little or as much as [you] like" (p. 440) and that they could ask for more of any of the items. After 15 minutes of being allowed to eat, the weight of snacks eaten by the child was estimated by weighing the snacks left over in each of the bowls and subtracting this from the weight of the food made available in that bowl: the weights of snacks eaten were then converted into energy values and totalled. This constituted the first dependent variable: snack energy intake.

Approximately 2 hours later, at lunchtime, each child was offered a lunch tray that included, on various days, food options described in the article as "vegetarian pizza," "chicken pieces,” "oven-baked chips,” “ vegetables,” "fruit,” and “yoghurt.” Again the children were told that they could ask for more of anything if they wished. Again, for each child, leftovers were weighed and subtracted from the weight of that food made available, and by summation the total energy value of the lunch food eaten was calculated. This constituted the second dependent variable: lunch energy intake. ${ }^{1}$

There are major design problems with this research and these will be discussed first, followed by problems with the data analysis and interpretation of the findings.

\section{MAJOR DESIGN PROBLEMS}

A detailed reading of Norman et al.'s Appetite article reveals at least eight major design problems that invalidate their study. These can be previewed as follows. First, Norman et al. did not do an adequate literature survey before planning the study. Second, the children were tested in an out-of-home leisure setting that likely introduced demand characteristics. Third, the children were exposed to an unrealistically heavy dose of advertising in the experiment. Fourth, the researchers neglected to include an unexposed control group and thus it could not be determined whether the children over-ate or under-ate following advertising exposure. Fifth, the children made their snack and lunch food choices in a likely peer-influenced group setting. Sixth, group-average calorie consumption, only, was reported, and these aggregate results hide the possibility of very different patterns of calorie intake. ${ }^{2}$ Seventh, the researchers neglected to interview the children at the

\footnotetext{
${ }^{1}$ This is a generous description of the researchers' procedure as given on p. 440 of their article. Numerous important details are missing which would prevent replication by other researchers. In particular there is a problem with the main dependent measure, snack energy intake. The researchers stated that each child "[was] given more of any food item on request," which suggests that extra food was provided beyond the food in the snack bowls in front of each child. If so, it is not clear how the researchers' measurement of food left over in the bowls could yield a measure of food consumed. Also, there is no mention of what beverages were provided to choose from; any beverage other than water, such as soft drink or fruit juice, would add calories.

${ }^{2}$ The author is indebted to an anonymous reviewer for making and numerically demonstrating this point.
} 
conclusion of the experiment to ask them what types of food they chose at snack time and why. Lastly, the question must be asked as to why the researchers believed that an experimental design involving a short intervention at a holiday camp would prove anything about the children's future likelihood of gaining weight. These problems are explained below.

\subsection{Inadequate literature survey for developing the research hypotheses}

The researchers overlooked the considerable early research conducted on the effects of advertising to children. Research on children's advertising issues, including the policy issue of whether to limit children's exposure to so-called junk-food advertising, dates back over five decades; see Adler, Lesser, Meringoff, Robertson, Rossiter, and Ward (1980) for a summary of the first decade of research and a discussion of associated policy issues that are still with us today. Two findings relevant to the Appetite study emerged from this early research. The first finding was that children's ability to detect persuasive intent in TV commercials develops steadily with age such that this ability is evident in all normal U.S. children by age 11 or 12 (Robertson \& Rossiter, 1974; Rossiter \& Robertson, 1976). A later review of research findings on this topic conducted for the American Psychological Association (Wilcox, Kunkel, Cantor, Dowrick, Linn, \& Palmer, 2004), also not cited by Norman et al., went further and concluded that children can reliably distinguish ads from programs by age 5 ; that by age 8 children realize that ads are trying to sell them something; and that by age 9 children become capable of detecting persuasive intent and that this ability is firmly established by age 11 to $12 .^{3}$ The second finding from the early research was that parental reports of their monitoring of their children's TV viewing are of doubtful honesty, with parents typically exaggerating their monitoring when compared with their own children's reports (Rossiter \& Robertson, 1975). Critics are likely to object that these studies were done with North American children, and almost 50 years ago. But it is unlikely that North American children's cognitive development is different from that of Australian children and not likely that children's advertising in the U.S. at that time differed much from children's advertising in Australia today.

What is also questionable about Norman et al.'s literature survey is that it was not disclosed that their study was an imitation of the earlier holiday camp experiment conducted by Gorn and Goldberg (1982) and published in the Journal of Consumer Research, an experiment prominently cited in the children's consumer research literature. ${ }^{4}$ Gorn and Goldberg's (1982) study is important for Norman et al. to have included because it supports their assumption - not actually tested in their later experiment ${ }^{5}$ - that "junk-food” TV

\footnotetext{
${ }^{3}$ Norman et al. included age as a covariate in their analysis (p. 440) but reported that age had no significant main effect and, more pointedly, had no significant interaction with snack energy consumption and exposure to junk-food advertising vs. non-food advertising. Perhaps the sample sizes at each age level were too small to detect any effect. Or perhaps, as argued later, other uncontrolled factors in their study washed out any effects of junk-food advertising.

${ }^{4}$ Although Norman et al. did cite Gorn and Goldberg's 1982 study in an article they published in another journal using the same data (see Norman, Kelly, McMahon, Boyland, Baur, Chapman, King, Hughes, \& Bauman, 2018b), they cited it only in passing, see p. 2, and did not disclose that they based their present study exactly on Gorn and Goldberg's earlier one. In their (2018a) Appetite article, they neglected to even cite the 1982 study but instead cited another article Gorn and Goldberg on children and TV advertising published in 1980 in the same journal, JCR. In the present author's opinion, these events could be considered to be a breach of research ethics.

${ }^{5}$ Norman et al., in contrast to Gorn and Goldberg, tested the hypothesis that TV advertising for so-called junkfood products would increase children's overall food, and thus energy, consumption - that is, that the
} 
advertising does increase children's unhealthy snack choices at a holiday camp. ${ }^{6}$

Specifically, Gorn and Goldberg found that in the experimental condition of a half-hour of children's cartoons, with nine 30-second “junk-food” TV commercials embedded, shown before afternoon snack time each day for the first 14 days of the camp, caused the children to choose candy over fruit on $75 \%$ of afternoon snack occasions. The control condition, administered to the same children in the following 14 afternoons of the camp, consisted of a half-hour of children's cartoons shown without any advertising, after which the children chose candy over fruit on $67 \%$ of afternoon snack occasions. There was therefore a statistically reliable extra $8 \%$ of candy choices attributable to junk-food advertising. Note, however, that the children in Gorn and Goldberg's study were between the ages of 5 and 8, too young to be reliably able to detect the ads' persuasive intent.

A consequence of Norman et al.’s limited literature search is that they formulated their hypotheses from a very narrow and biased foundation, namely, their own previous questionable studies ${ }^{7}$ or related questionable studies by other health researchers. ${ }^{8}$ The main hypothesis that Norman et al. quite obviously set out to test was that children's exposure to TV advertising for junk-food products would cause them to overeat. Perhaps because this hypothesis was not upheld, the researchers relied on two supplementary hypotheses about parental rules governing their children's eating. The first supplementary hypothesis predicted that parents who restrict their children's diet at home (e.g., p. 440: "If I did not guide or regulate my child's eating he/she would eat too much of their favorite foods") would cause the child, apparently in a sort of reactionary response, to overeat at the holiday camp after exposure to junk-food TV advertising. The second supplementary hypothesis, however, seems to contradict the first, predicting that parents who encourage their children to overeat at home (e.g., p. 440: "My child should always eat all the food on his/her plate") also would cause the child to overeat at the camp after exposure to junk-food TV advertising. This second hypothesis is hardly plausible because prolonged forced eating, far from causing overeating, would tend to have the opposite effect of rendering food cues in ads and food presented at snack time aversive.

\subsection{Demand characteristics}

Norman et al. claimed that a holiday camp without parents present is the "ideal environment” to test these hypotheses (p. 439). The holiday camp setting, however, represents an unusual and different stimulus context from both the normal in-home eating situation and from the typical out-of-home eating situation. The normative rule in holiday

advertising would cause children to overeat. Although they obviously had the data, Norman et al. neglected to test the policy-relevant hypothesis that such advertising would cause children to overeat junk food.

${ }^{6}$ Gorn and Goldberg (1982) also found that anti junk-food TV advertising was somewhat effective, though not as expected. In their experiment, fruit industry commercials reduced children's choice of Kool-Aid over water as a beverage but did not significantly reduce their choice of candy over fruit. Disappointingly for public health advocates, government-sponsored TV public service announcements (PSA commercials) for healthy eating did not reduce children's choice of either candy over fruit, or Kool-Aid over water.

\footnotetext{
${ }^{7}$ The present author checked most of Norman's co-authors' previous research publications on this policy-related topic and is not just making an uninformed comment. Space does not permit detailed criticism of their earlier work, other than to point out the paucity of attention they gave to the marketing and consumer behavior literature.

${ }^{8}$ Two of these studies, which Norman et al. used to select the measures for testing their parental control hypotheses, were published in Appetite: Birch et al. (2001) and Vollmer and Baietto (2017). However, the focus is on the present Appetite study and spaces not permit criticism of these studies here.
} 
settings is "eat what and as much as you like." This rule - a demand characteristic, in effect - would normally be offset in a supervised situation such as a school outing, for example, but in the holiday camp situation the researchers openly encouraged splurging or overeating by telling the children that they could eat as little or as much as they liked and could ask for more if they wished (p. 440). This may have worked against the researchers' main hypothesis because it could be that the "holiday splurge" demand characteristic was so strong that it overrode any difference in calorie consumption caused by exposure to junk-food advertising vs. non-food advertising.

Another demand characteristic, too, may have worked against the researchers' main hypothesis. This is the fact that the researchers employed, as noted, a crossover experimental design in which all the children were exposed to TV advertising for allegedly unhealthy food products. This may have signalled to the children, in a demand characteristic manner, that the holiday camp was supportive of these products and may even have been promoting them.

\subsection{Unrealistically heavy dose of advertising}

The researchers embedded 5 minutes of advertising - ten 30-second commercials - in a 10-minute cartoon. The Australian Communications and Media Authority's (2014) government regulatory code in Australia allows a maximum of 10 minutes of advertising per hour in children's programs, which translates to $1 \mathrm{~min} 40 \mathrm{sec}$ of advertising in 10 minutes of programming. This means that the 5 minutes of advertising shown to the children is treble the self-regulatory limits. Moreover, attention was obliged if not forced, and the same 10 commercials were shown every day for three days in a row. This unrealistically heavy dosage poses a major generalizability problem. ${ }^{9}$

The other children randomly selected into the multi-media advertising group were additionally told to play a TV-like advergame for 5 minutes that, on the junk-food advertising days, featured a branded "unhealthy" food product throughout. If the game did not forewarn the children that this brand was the sponsor of the game, thereby warning them that the whole advergame was an advertisement, then this constitutes an unethical product placement contrary to industry regulations. ${ }^{10}$ In any case, the addition of the advergame contributes to the atypically heavy dose of advertising that the children in the multimedia group were exposed to.

\subsection{No unexposed control group or report of absolute energy intake}

As will be seen shortly, Norman et al. reported only the difference in energy (kilojoules) consumed by the children on the junk-food advertising days and the non-food advertising days instead of reporting the absolute amounts of energy consumed. Norman et al.'s main hypothesis was that children would consume more calories than they would normally at morning snack time - that is, overeat - after exposure to junk-food advertising. However, to test whether there was overeating, there would have to have been a separate control condition of days on which the children were not exposed to any media before morning snack time. Absolute calories consumed on the junk-food advertising days, on the

\footnotetext{
${ }^{9}$ The only similar situation in which such heavy advertising exposure might occur is with pre-Christmas advertising for toys and games. See Robertson and Rossiter (1976).

${ }^{10}$ Unannounced product placements are banned in all westernized countries, with the exception of the U.S.A. And specific to the present study, the self-regulatory guidelines of the Australian Food and Grocery Council (2014) allow product placements in children's interactive games only if the food or beverage products featured is classified as nutritionally healthy.
} 
non-food advertising days, and on the no advertising days could then be compared to see whether overeating occurred.

Another possibility not able to be ruled out without a no-exposure control condition is that the cartoons, and in the multi-media group the advergame that followed, caused such high arousal in the children watching and playing that it washed out any differences caused by the type of advertising that was shown. Zillmann's (1978, 1982; see also Rossiter \& Foxall, 2008) "excitation-transfer theory," an extension of the Hullian performance equation of drive $\times$ habit strength, would predict that with high arousal carryover from watching the cartoons and perhaps playing the advergame, the eating response would be so strong as to override any effect of the advertising.

\subsection{Group influence on food choice}

The children made their food choices in a group setting - at a snack table and a lunch table in sight of other children. The likelihood of influence from popular peers and the likelihood of older children's visible choices influencing the choice of the younger ones is very great, especially in a novel setting where the more anxious or younger children may not be sure what and how much to eat. Whereas it could be argued that the social influence would apply equally across the experimental conditions, chances are that it would also wipe out the differences between them and thus invalidate the experiment.

\subsection{Group results mask individual consumption patterns}

As will be seen shortly, the researchers reported their findings on the children's calorie consumption as a group average. This aggregate analysis assumes every individual child starts from the same baseline of calorie consumption and behaves similarly in response to the food advertising and non-food advertising interventions. The individual children's consumption patterns should have been measured and the average and standard deviation of these reported. Better still, the researchers could have reported the number and percentage of children showing an increase, a decrease, or no change in calorie consumption after food advertising and after non-food advertising.

\subsection{No questioning of the children afterwards}

One may well ask why the researchers did not, at the end of the experiment, ask the children what they chose at snack time - and why. This could have been done at the end of the sixth and final day and the children could have been asked only about which snack food, if any, they chose earlier that day (recall that half the children would have finished the experiment on a junk-food advertising day and the other half would have finished on a nonfood advertising day). Even 10 interviews per condition, or about 40 total out of the total of 78 children who completed the experiment, would have been sufficient to obtain a reasonable indication of snack food choices and the children's reasons for them, as well as possibly providing evidence of whether exposure to food advertising vs. non-food advertising made any difference.

Open-ended questioning of the children at the conclusion of the camp might also have spontaneously brought out the nature and influence of any parental rules about eating. According to previous research (Rossiter \& Robertson, 1975), the children's reports of parental supervision would be far more trustworthy than the typically exaggerated parental reports.

\subsection{Speculation from a temporary intervention with mostly normal-weight children}


The final criticism concerns the researchers' speculation that a short intervention in an away-from-home setting would shed light on the causes of the childhood obesity problem. As reported in Table 1 on p. 441 of their article, $80 \%$ of the children were of normal weight according to the BMI measure, and a finding that mostly normal-weight children splurge a little at holiday camp means little. And the $16 \%$ overweight or obese children may have overeaten anyway. But did their exposure to junk-food advertising make any difference? To answer this last question, we turn to the actual findings.

\section{THE STUDY'S FINDINGS}

In the present author's long experience with editors and journal reviewers, many seem to be under the impression that if the empirical results support the hypotheses, then methodological faults can be forgiven or overlooked. This is not, of course, good science. If there are methodological faults - and before that, mistakes in the theoretical arguments leading to the hypotheses - then the empirical findings do not matter and the paper should be rejected. As amply demonstrated above, this is the case with Norman et al.'s findings. It is, however, worth examining their findings if only to show that regardless of the foregoing problems the findings do not support their case against junk-food advertising.

Norman et al.'s findings are reproduced in the present Table 1. The present writer has translated the energy measure to calories to prevent the "gee whiz" effect that results from the enormously high numbers that the kilojoule measure produces. It should be pointed out firstly that the researchers wrongly went beyond snack-time calorie consumption and looked also at lunch-time calorie consumption, and then summed calorie consumption over the two eating opportunities. This is not legitimate because obviously if unhealthy food advertising was to have an effect on calorie consumption it should have occurred at snack time or not at all; it is simply not credible for the researchers to argue that there could be no effect at snack time but then a delayed effect at lunchtime. Accordingly, the present table of their findings shows only the calorie consumption differences at snack time: the difference between calories consumed at snack time on the junk-food advertising days and calories consumed at snack time on the non-food advertising days.

Table 1 about here

To provide evidence in support of a ban on children's junk-food advertising, Norman and her senior colleagues would have had to have shown that the children's exposure to TV advertising for such products in the experiment, rather than such exposure boosted by playing the junk-food-featuring advergame, resulted in an increase in calories consumed at snack time. However, as can be seen in the upper panel of the table, exposure to TV advertising for allegedly unhealthy food products did not cause an increase in the children's calorie consumption - an increase of only 5 calories after exposure is not significantly different from a zero effect. As can be seen in the lower panel, it took the addition of the unhealthy food product placement in the advergame to produce a significant difference in favor of the junkfood advertising hypothesis - but this difference was trivial in that 48 calories is an increase of only $2 \%$ of the recommended daily calorie intake. As footnoted in the table, 48 calories is equivalent to drinking about one-third of a small (300 mil.) bottle of regular Coke or taking one bite out of a Cherry Ripe chocolate bar.

Norman et al.'s tests of the supplementary hypotheses about parental control also did not turn out well. Recall that the researchers hypothesized firstly an interaction between parents' restriction of their children's eating at home and exposure to unhealthy food advertising at the camp such that parentally restricted children would eat more calories afterwards, a hypothesis which was not supported in the TV advertising group and was not 
supported in the TV advertising-plus-advergame group either. The researchers hypothesized secondly an interaction between parental encouragement at home to finish their food and exposure to unhealthy food advertising at the camp such that, strangely, these force-fed children, too, would overeat afterwards, a hypothesis that was not supported in the TV advertising group and received only slight support in the TV advertising-plus-advergame group.

It is inappropriate, therefore, for the researchers to conclude that their findings prove a relationship between junk-food TV advertising and children's tendency to overeat. Nor can Norman et al. draw any sound conclusions about the parental control of their children's eating behavior given the doubtful veracity of parental reports.

\section{DISCUSSION AND RECOMMENDATIONS}

A serious problem with Norman et al.'s reporting in the Appetite study is that it fails to refer to the current Australian government regulations and industry guidelines about advertising directed to children. Norman et al. called for "more stringent regulatory policy to restrict children's exposure to unhealthy food marketing” (p. 442) without disclosing that the current Australian government policy, as specified by the Australian Communications and Media Authority (2014) under the Broadcasting Services Act of 1992, is already restrictive. The government's ACMA policy bans all advertising during preschool children's " $\mathrm{P}$ ” TV programs. The ACMA policy limits advertising time to 5 minutes per half-hour before, during, and after children's " $C$ ” programs (those designed for children in the age group 5 to 13), whereas Norman et al. showed 5 minutes of advertising in just 10 minutes of programming. Not credited by Norman et al. is the fact that the government's ACMA policy lists at least 10 restrictions on children's advertising content - restrictions designed to ensure, among other things, that all ads must be clearly distinguished from programming, that product claims are not intentionally deceptive or unintentionally misleading or ambiguous, that children's ads do not use celebrity endorsements of any kind, and that such ads do not "pressure" parents, or anyone else, to buy the advertised product for the child. The advertising industry's self-regulatory codes (Australian Association of National Advertisers, 2014, 2015) comply with all these restrictions. Also, while the government regulations apply only to TV advertising, the Australian food industry's self-regulatory codes for marketing to children (Australian Food and Grocery Council, 2014, 2018) prohibit food and beverage product placements in children's TV programs and on the Internet, which would cover advergames, meaning that Norman et al.'s multi-media condition violated this code. The government regulations and the self-regulatory codes do not apply to parent-directed ads for products that children may consume or use, and they do not apply to child-directed ads that are placed outside the C-program advertising times of 7 a.m. to 8:30 a.m. and 4 p.m. to 8:30 p.m. weekdays, and weekends and school holidays from 7 a.m. to 8:30 p.m., recognizing that child-directed ads are rarely deliberately placed outside these times.

It is also good scientific practice to consider a counter-hypothesis when testing one's own hypothesis. William J. McGuire's $(1964,1969)$ inoculation hypothesis implies that it is actually counterproductive to prevent children's exposure to advertising. ${ }^{11}$ The inoculation hypothesis would predict that children's exposure to TV commercials is necessary in order for children to build their cognitive defenses (Rossiter \& Robertson, 1974) and thus allow

\footnotetext{
${ }^{11}$ McGuire's well-known inoculation hypothesis was discussed in Adler et al.'s (1980) review of children's advertising research and policy but this earlier work was inexplicably overlooked in Wilcox et al.'s (2004) review conducted for the American Psychological Association. Norman et al.'s limited literature search found neither.
} 
them to become "immunized" against persuasion. The ideal situation for immunization, of course, is for parents to watch children's programs with them and comment critically, if need be, when the commercials come on. However, the previous research not cited in Norman et al.'s article would suggest that parental education about persuasion would not have much real effect until the child reaches the age of about 9 and begins to genuinely comprehend advertising's persuasive intent. With children of all ages, of course, parents should be responsible for deciding whether or not to accede to children's requests for advertised products.

Whereas the present critique would seem to be about just one empirical study, albeit a study approved by experienced social science researchers, its implications are much broader. One must question now such obviously flawed studies get to the journals in the first place. Here, the blame must lie mainly with the low level of research training available in the social sciences, at least in Australian universities, and thus the poor vetting of research proposals by government grant-giving institutions. In the present author's opinion, all proposed research studies should be required to pass the scrutiny of several multidisciplinary seminars preferably at different universities - attended by other researchers unconnected with the study and attended by all the faculty's research students, with attendees instructed beforehand to be as critical as possible during the presentation. Now, with several universities conveniently located in the same city, this requirement could easily be introduced in Australia. Indeed, if introduced worldwide this critical multidisciplinary scrutiny would go a long way towards reducing the flood of questionable studies appearing in our journals. This pre-research vetting is particularly important if the research is intended to be an input to legislation.

Lastly, in relation to Norman et al.'s study and to the several other "anti-advertising" studies that have been published about childhood obesity, there is a very basic question to be answered. If there was no significant child obesity problem in the pre-regulation era when children were exposed to more "junk food" advertising than they are today, how can today's junk-food advertising possibly be blamed for today's problem? Also, as pointed out by Alison Watkins (2018), group managing director of Coca-Cola Amatil, trend figures from the Australian Bureau of Statistics show that over the past decade or so, since 1995, per capita consumption of $\operatorname{sugar}^{12}$ from foods and beverages has fallen $6 \%$ among adults and fallen a substantial 23\% among children, while over the same period child-directed TV advertising for allegedly unhealthy foods and beverages has remained relatively constant. The blame for children's weight gain and childhood obesity therefore must lie not with junk-food advertising but with parents. ${ }^{13}$ Too many parents are failing to limit their children's calorie intake and commensurately failing to ensure that their children are getting enough physical exercise.

\section{REFERENCES}

\footnotetext{
12 Ironically enough, urging Australians to eat more fruit and vegetables will result in increased consumption of fructose, the natural sugar in fruit, which experts claim (see, e.g., Skerret, 2011) to be much more harmful to health than sucrose, most likely because fructose does not neurologically signal satiety nearly as well as sucrose does, meaning that the fruit-juice drinker is likely to want to consume more calories afterwards.

13 This is also apparently the view of the Australian Medical Association. A spokesperson from the AMA, citing a recent study from Harvard, said that "mums who role-modelled healthy physical and nutritional habits were less likely to have children with weight problems...[and] 75 per cent less likely to have obese kids" (Lawrence, 2018, p.11).
} 
Adler, R.P., Lesser, G.S., Meringoff, L.K., Robertson, T.S., Rossiter, J.R., \& Ward, S. (1980). The effects of television advertising on children. Lexington, MA: Lexington Books.

Australian Association of National Advertisers (2014). Code for Advertising \& Marketing Communications to Children. www.aana.com.au

Australian Association of National Advertisers (2015). Practice Note to the Code of Advertising and Marketing Communications to Children. www.aana.com.au

Australian Communications and Media Authority (2014). Children's Television Standards 2009. www.acma.gov.au

Australian Communications and Media Authority (2015). Time-limit rules for advertising on TV. www.acma.gov.au

Australian Food and Grocery Council (2014). Quick Service Restaurant Initiative for Responsible Advertising and Marketing to Children. www.afgc.org.au

Australian Food and Grocery Council (2016). Responsible Children’s Marketing Initiative. www.afgc.org.au

Birch, L.L., Fischer, J.O., Grimm-Thomas, K., Markey, C.N., Sawyer, R, and Johnson, S.L. (2001). Confirmatory factor analysis of the Child Feeding Questionnaire: A measure of parental attitudes, beliefs and practices about child feeding and obesity proneness. Appetite, 36, 201-210.

Gorn, G.J., \& Goldberg, M.E. (1982). Behavioral evidence of the effects of televised food messages on children. Journal of Consumer Research, 9, 200-205.

Griscti, C. (2018). “Is it time to give junk food ads the flick?” The Sunday Telegraph, July 8, body + soul magazine, pp. 4-5.

Lawrence, E. (2018). “Exercise the full force of the law.” The Daily Telegraph, September 8, p. 11.

McGuire, W.J. (1964). Inducing resistance to persuasion. In L. Berkowitz (Ed.), Advances in experimental social psychology (Vol. 1, pp. 191-229). New York: Academic Press.

McGuire, W.J. (1969). The nature of attitudes and attitude change. In G. Lindsey \& E. Aronson (Eds.), The handbook of social psychology (second edition, Vol. 3, pp. 136314). Reading, MA: Addison-Wesley.

Monafo, M.R., Nosek, B.A., Bishop, D.V.M., Button, K.S., Chambers, C.D., Percie du Sert, N., Simonsohn, U., Wagenmakers, E-J., Ware, J.J., \& Ioannidis, J.P.A. (2017). A manifesto for reproducible science. Nature Human Behaviour, 1, article no. 0021, 1-9.

Norman, J., Kelly, B., McMahon, A-T., Boyland, E., Baur, L.A., Chapman, K., King, L., Hughes, C., \& Bauman, A. (2018a). Children's self-regulation of eating provides no defense against television and online food marketing. Appetite, 125, 438-444.

Norman, J., Kelly, B., McMahon, A-T., Boyland, E., Baur, L.A., Chapman, K., King, L., Hughes, C., \& Bauman, A. (2018b). Sustained impact of energy-dense TV and online food advertising on children's dietary intake: A within-subject, randomised, crossover, counter-balanced trial. International Journal of Behavioral Nutrition and Physical Activity, 15, e1-e11. 
Parnell, S. (2018). “Obesity worries fail to hit the sweet spot.” The Australian, June 1, p. 12.

Robertson, T.S., \& Rossiter, J.R. (1974). Children and commercial persuasion: An attribution theory analysis. Journal of Consumer Research, 1 (1), 13-20.

Robertson, T.S., \& Rossiter, J.R. (1976). Short-run advertising effects on children: A field study. Journal of Marketing Research, 13, 68-70.

Rossiter, J.R., and Foxall, G.R. (2008). Hull-Spence behavior theory as a paradigm for consumer behavior. Marketing Theory, 8 (2), 123-141.

Rossiter, J.R., \& Robertson, T.S. (1974). Children's TV advertising: Testing the defenses. Journal of Communication, 24, 137-144.

Rossiter, J.R., \& Robertson, T.S. (1975). Children's television viewing: An examination of parent-child consensus. Sociometry, 38 (2), 308-326.

Rossiter, J.R., \& Robertson, T.S. (1976). Canonical analysis of developmental, social, and experiential factors in children's comprehension of television advertising. Journal of Genetic Psychology, 129 (2), 317-327.

Skerrit, P.J. (2011). Is fructose bad for you? www.health.harvard.edu/blog/is-fructose-badfor-you- 201104262425

The Economist (2018). “Dodgy peer review disrupts quality control.” Reproduced in The Australian, Higher Education, June 29, p. 32.

Vollmer, R.L., \& Baietto, J. (2017). Practices and preferences: Exploring the relationships between food-related parenting practices and child food preferences for high fat and/or sugar foods, fruits, and vegetables. Appetite, 113, 134-140.

Watkins, A. (2018). “Sugar tax is no cure for obesity.” The Australian, May 7, p. 12.

Wilcox, B.L.,Kunkel, D., Cantor, J. Dowrick, P., Linn, S., \& Palmer, E. (2004). Report of the APA Task Force on Advertising and Children. Washington, DC: American Psychological Association.

Zillmann, D. (1978). Transfer of excitation in emotional behavior. In J.T. Cacioppo \& R.E. Petty (Eds.), Social psychophysiology (pp. 215-240). New York: Guilford Press.

Zillmann, D. (1982). Television viewing and arousal. In D. Pearl, L. Bouthilet. \& J. Lazar (Eds.), Television and behavior: Ten years of progress and implications for the eighties. Washington, DC: U.S. Government Printing Office. 
Table 1 Norman et al.'s (2018) results measured in calories. Entries show the difference (unhealthy food advertising condition minus non-food advertising condition) in snack-food calories consumed immediately after exposure to the ads.

Advertising exposure groups - TV vs. TV + Advergame - and parental control subgroups (sample and subsample sizes in parentheses)
Snack-food calorie consumption difference scores (asterisked entries show the statistically significant differences at $5 \%$ or $1 \%)$

\begin{tabular}{lr} 
TV ads & \\
\hline All children $(\mathrm{N}=76)$ & +5 \\
\hline Restricted eating $(\mathrm{n}=36)$ & -11 \\
No restriction $(\mathrm{n}=40)$ & -17 \\
\hline Encouragement to eat $(\mathrm{n}=19)$ & +12 \\
No encouragement $(\mathrm{n}=57)$ & +2 \\
\hline
\end{tabular}

\begin{tabular}{ll} 
TV ads + Advergame & \\
\hline All children $(\mathrm{N}=78)$ & $+48^{*}$ \\
\hline Restricted eating $(\mathrm{n}=45)$ & +38 \\
No restriction $(\mathrm{n}=33)$ & +61 \\
\hline Encouragement to eat $(\mathrm{n}=20)$ & $+85^{* *}$ \\
No encouragement $(\mathrm{n}=58)$ & +35 \\
\hline
\end{tabular}

\footnotetext{
*To put this difference into perspective, +48 calories is equivalent to eating one-fifth of a regular size Cherry Ripe chocolate bar, or drinking one-third of a small bottle of Classic Coke. The 48 calorie difference is only about $2 \%$ of the recommended daily intake of calories, or energy.

**The difference of +85 calories is equivalent to one-third of a regular Cherry Ripe bar, or $60 \%$ of a small bottle of Classic Coke, and is only about $4 \%$ of the recommended daily intake of energy. But the difference of +35 calories for the no-pressure children should be subtracted from that, leaving only a +50 calorie relative increase in energy consumption, or $2 \%$ of the recommended daily energy intake, among the pressure-to-eat children.
} 
